\title{
An Improved Measurement-Oriented Marginal Multi-Bernoulli/Poisson Filter
}

\author{
Zhen-zhen SU, Hong-bing JI, Yong-quan ZHANG \\ School of Electronic Engineering, Xidian University, Xi'an 710071, People’s Republic of China \\ zzsu@stu.xidian.edu.cn, hbji@xidian.edu.cn, zhangyq@xidian.edu.cn \\ Submitted March 4, 2018 / Accepted November 11, 2018
}

\begin{abstract}
The measurement-oriented marginal multi-Bernoulli/Poisson $(M O M B / P)$ filter is an attractive approach for multi-target tracking. However, the effect of measurement on predicted target states may be weakened when the hypothesized tracks are separated, even if the measurement is close to the predicted target state. This is due to the inaccuracy of the missed detection hypothesis probabilities in the marginal association probabilities. To solve this problem, an improved $M O M B / P(I M O M B / P)$ filter is proposed in this paper, by considering the measurement information in the missed detection hypotheses. Simulation results reveal a favorable comparison to the $M O M B / P$ filter in terms of the Optimal Subpattern assignment (OSPA) distance and cardinality estimation.
\end{abstract}

\section{Keywords}

Multi-target tracking, random finite set, $\mathrm{MOMB} / \mathrm{P}$ filter, missed detection hypothesis

\section{Introduction}

Recently, random finite set (RFS) theory has attracted extensive applications in multi-target tracking (MTT) [1], [2]. Some RFS-based filters have been proposed, such as the probability hypothesis density (PHD) filter [3], the cardinalized PHD (CPHD) filter [4], the multi-target multiBernoulli (MeMBer) filter [2] and the cardinality-balanced MeMBer (CBMeMBer) filter [5]. Even though the combinatorial problems caused by data association in the PHD and CPHD filters can be avoided, a potentially unreliable clustering step is usually required in the sequential Monte Carlo (SMC) implementation [6], [7]. By using multi-Bernoulli representation in the MeMBer and CBMeMBer filters, reliable and inexpensive extraction of state estimates can be achieved. But note that they are only appropriate for the cases with low clutter density [5].

In Bayesian probability theory, the prior distribution is a conjugate-prior form for the likelihood function, which means that the prior distribution has the same form with the posterior distribution. The conjugacy is an attractive prop- erty with an exact posterior density. Thus, some conjugate priors based filters [8],[13] have been proposed, which use a specific mixture of multi-Bernoulli probability density functions to represent the posterior multi-target probability density function (pdf). The generalized labeled multiBernoulli (GLMB) filter [9], [10] is based on the labeled multi-Bernoulli conjugate prior with the label information of targets. To reduce the computational complexity, an extended the GLMB filter was proposed in [11]. In addition, two marginal multi-Bernoulli filters were proposed in [12], namely the measurement-oriented marginal multi-Bernoulli/Poisson (MOMB/P) filter and the target-oriented marginal multi-Bernoulli/Poisson (TOMB/P) filter. The MOMB/P and TOMB/P filters are based on Poisson multi-Bernoulli mixture (PMBM) conjugate prior. In [14], the sequential Monte Carlo implementation of the TOMB/P filter has been proposed. The PMBM conjugate prior based multiple extended target tracking filter was proposed in [15].

The marginal multi-Bernoulli filters assume that the global association probabilities are factorized into the marginal association probabilities. Then, the marginal association probabilities can be calculated by the loopy belief propagation (LBP) algorithm with less computational complexity. Actually, the MOMB/P filter is a combination of the MeMBer filter and LBP data association approximation, which is robust to coalescence and can achieve better performance than the CPHD and CBMeMBer filters do [12].

Although the MOMB/P filter has excellent performance, it exhibits lower performance when targets are well separated [13]. This is because the cardinality estimation of the $\mathrm{MOMB} / \mathrm{P}$ filter is inaccurate when the marginal association probability has error. In the marginal association probability, the effect of measurement on predicted target state may be weakened, even if the measurement is close to the predicted target state, since the measurement information is not involved in the missed detection hypotheses. To solve this problem, an improved MOMB/P (IMOMB/P) filter is proposed with more accurate cardinality estimation by considering the measurement information in the missed detection hypotheses. Simulations show that the proposed filter outperforms the $\mathrm{MOMB} / \mathrm{P}$ filter in a challenging scenario. 
The rest of this paper is organized as follows. In Sec. 2, the MOMB/P filter is reviewed and the existing problems are presented. Then the IMOMB/P filter is proposed in Sec. 3. Section 4 presents the simulation results, subsequently conclusion is drawn in Sec. 5.

\section{Background}

This section involves multi-target system model, the $\mathrm{MOMB} / \mathrm{P}$ filter and its problem description.

\subsection{Multi-Target System Model}

In this paper, the standard system model [1], [3] is used for MTT with measurement origin uncertainty. At time $k, N_{k}$ target states are denoted by $\mathbf{x}_{k}{ }^{1}, \ldots, \mathbf{x}_{k}{ }^{N_{k}} \in \mathbb{R}^{n_{x}}$ consisting of parameters such as position, velocity, etc. Meanwhile, a sensor observes $M_{k}$ measurements $\mathbf{z}_{k}{ }^{1}, \ldots, \mathbf{z}_{k}^{M_{k}} \in \mathbb{R}^{n_{z}}$. The target states and measurements are treated as the multi-target state and multi-target measurement respectively, i.e.,

$$
\begin{aligned}
& \mathbf{X}_{k}=\left\{\mathbf{x}_{k}^{1}, \cdots, \mathbf{x}_{k}^{N_{k}}\right\}, \\
& \mathbf{Z}_{k}=\left\{\mathbf{z}_{k}^{1}, \cdots, \mathbf{z}_{k}^{M_{k}}\right\} .
\end{aligned}
$$

Using RFS theory, the time evolution of the multitarget state and the multi-target measurement can be constructed as follows.

At time $k-1$ a target with state $\mathbf{x}_{k-1}$ continues to exist at time $k$ with probability $p_{s}\left(\mathbf{x}_{k-1}\right)$ and moves to a new state $\mathbf{x}_{k}$, or dies with probability $1-p_{s}\left(\mathbf{x}_{k-1}\right)$. Thus, at time $k$ the target with state $\mathbf{x}_{k-1}$ is modeled as a Bernoulli RFS $\mathbf{S}_{k}\left(\mathbf{x}_{k-1}\right)$ with existence probability $p_{s}\left(\mathbf{x}_{k-1}\right)$ and spatial pdf $f\left(\mathbf{x}_{k} \mid \mathbf{x}_{k-1}\right)$. Assuming that the targets are mutually independent, the multi-target state $\mathbf{X}_{k}$ at time $k$ is given by the union [1], [3]

$$
\mathbf{X}_{k}=\left[\bigcup_{x_{k-1} \in X_{k-1}} \mathbf{S}_{k}\left(\mathbf{x}_{k-1}\right)\right] \cup \boldsymbol{\Gamma}_{k}
$$

where $\boldsymbol{\Gamma}_{k}$ denotes the newborn targets which is a Poisson RFS with mean $\mu_{b}$, spatial pdf $b\left(\mathbf{x}_{k}\right)$ and intensity function $\lambda_{b}\left(\mathbf{x}_{k}\right)=\mu_{b} b\left(\mathbf{x}_{k}\right)$. This model defines the RFS transition pdf $f\left(\mathbf{X}_{k} \mid \mathbf{X}_{k-1}\right)$.

An existing target with state $\mathbf{x}_{k}$ is detected with probability $p_{\mathrm{d}}\left(\mathbf{x}_{k}\right)$ and generates a measurement $\mathbf{z}_{k}$ with spatial $\operatorname{pdf} f\left(\mathbf{z}_{k} \mid \mathbf{x}_{k}\right)$, or missed with probability $1-p_{\mathrm{d}}\left(\mathbf{x}_{k}\right)$. In addition, measurements may be generated by clutter or false alarms which can be modeled as a Poisson RFS $\mathbf{K}_{k}$ with mean $\mu_{\mathrm{c}}$, spatial pdf $f_{c}\left(\mathbf{z}_{k}\right)$ and intensity function $\lambda_{c}\left(\mathbf{z}_{k}\right)=\mu_{c} f_{c}\left(\mathbf{z}_{k}\right)$. Thus, the multi-target measurement $\mathbf{Z}_{k}$ at time $k$ is given by the union [1], [3]

$$
\mathbf{Z}_{k}=\left[\bigcup_{x_{k} \in X_{k}} \boldsymbol{\Theta}_{k}\left(\mathbf{x}_{k}\right)\right] \cup \mathbf{K}_{k} .
$$

This model defines the RFS likelihood function $f\left(\mathbf{Z}_{k} \mid \mathbf{X}_{k}\right)$.

\subsection{Review of the MOMB/P Filter}

Filters based on RFS consist of prediction step and update step. In prediction step, the previous posterior pdf $f\left(\mathbf{X}_{k-1} \mid \mathbf{Z}_{1: k-1}\right)$ is converted into the predicted pdf $f\left(\mathbf{X}_{k} \mid \mathbf{Z}_{1: k-1}\right)$ involving the transition pdf $f\left(\mathbf{X}_{k} \mid \mathbf{X}_{k-1}\right)$. In update step, the predicted pdf $f\left(\mathbf{X}_{k} \mid \mathbf{Z}_{1: k-1}\right)$ is converted into $f\left(\mathbf{X}_{k} \mid \mathbf{Z}_{1: k}\right)$ involving the likelihood function $f\left(\mathbf{Z}_{k} \mid \mathbf{X}_{k}\right)$. Note that, in different filters, different assumptions and approximations are used to reduce the computational complexity.

In this section, the $\mathrm{MOMB} / \mathrm{P}$ filter in [12] is reviewed. The posterior multi-target $\operatorname{pdf} f\left(\mathbf{X}_{k} \mid \mathbf{Z}_{1: k}\right)$ is given by the convolution [1]

$$
f\left(\mathbf{X}_{k} \mid \mathbf{Z}_{1: k}\right)=\sum_{\mathbf{Y} \subseteq \mathbf{X}_{k}} f_{u}(\mathbf{Y}) f_{d}\left(\mathbf{X}_{k} \backslash \mathbf{Y}\right)
$$

where $\mathbf{X}_{k} \backslash \mathbf{Y}$ is the set of elements in $\mathbf{X}_{k}$ but not in $\mathbf{Y}$, $f_{u}\left(\mathbf{X}_{k}\right)$ is the pdf of unknown targets $\mathbf{X}_{k}^{u}$ and $f_{d}\left(\mathbf{X}_{k}\right)$ is the posterior pdf of detected targets $\mathbf{X}_{k}^{d}$. Furthermore, $\mathbf{X}_{k}^{u}$ is modeled as a Poisson RFS with mean $u_{u}$, spatial pdf $f_{u}\left(\mathbf{x}_{k}\right)$ and intensity function $\lambda_{u}\left(\mathbf{x}_{k}\right)=u_{u} f_{u}\left(\mathbf{x}_{k}\right) . \mathbf{X}_{k}^{d}$ is modeled as a multi-Bernoulli RFS involving $I_{k}$ Bernoulli components, each composes existence probability $r_{k}^{i}$ and spatial pdfs $s_{k}^{i}\left(\mathbf{x}_{k}\right)$. Then, the MOMB/P filter is given as follows including the prediction step and the updating step.

During the prediction step, the intensity function $\lambda_{k \mid k-1}^{u}\left(\mathbf{x}_{k}\right)$ of $f_{k \mid k-1}^{u}\left(\mathbf{X}_{k}\right)$ is given by

$$
\lambda_{k \mid k-1}^{u}\left(\mathbf{x}_{k}\right)=\lambda_{b}\left(\mathbf{x}_{k}\right)+\int f\left(\mathbf{x}_{k} \mid \mathbf{x}_{k-1}\right) p_{s}\left(\mathbf{x}_{k-1}\right) \lambda_{u}\left(\mathbf{x}_{k-1}\right) \mathrm{d} \mathbf{x}_{k-1}
$$

where $\lambda_{u}\left(\mathbf{x}_{k-1}\right)$ is the intensity function of $f_{u}\left(\mathbf{X}_{k-1}\right) \cdot r_{k \mid k-1}^{i}$ and $s_{k \mid k-1}^{i}\left(\mathbf{x}_{k}\right)$ of $f_{k \mid k-1}^{d}\left(\mathbf{X}_{k}\right)$ are given by

$$
\begin{gathered}
r_{k \mid k-1}^{i}=r_{k-1}^{i} \int p_{s}\left(\mathbf{x}_{k-1}\right) s_{k-1}^{i}\left(\mathbf{x}_{k-1}\right) \mathrm{d} \mathbf{x}_{k-1}, \\
s_{k \mid k-1}^{i}\left(\mathbf{x}_{k}\right)=\frac{\int f\left(\mathbf{x}_{k} \mid \mathbf{x}_{k-1}\right) p_{s}\left(\mathbf{x}_{k-1}\right) s_{k-1}^{i}\left(\mathbf{x}_{k-1}\right) \mathrm{d} \mathbf{x}_{k-1}}{\int p_{s}\left(\mathbf{x}_{k-1}\right) s_{k-1}^{i}\left(\mathbf{x}_{k-1}\right) \mathrm{d} \mathbf{x}_{k-1}}
\end{gathered}
$$

for $i \in\left\{1, \cdots, I_{k-1}\right\}$, where $r_{k-1}^{i}$ and $s_{k-1}^{i}\left(\mathbf{x}_{k-1}\right)$ are parameters in $f_{d}\left(\mathbf{X}_{k-1}\right)$. The number of Bernoulli components $I_{k-1}$ does not change in the prediction step, so does the number of unknown target hypotheses $I_{k-1}^{u}$ (in the linear Gaussian implementation).

During the update step, the intensity function $\lambda_{u}\left(\mathbf{x}_{k}\right)$ of $f_{u}\left(\mathbf{X}_{k}\right)$ is given by

$$
\lambda_{u}\left(\mathbf{x}_{k}\right)=\left(1-p_{d}\left(\mathbf{x}_{k}\right)\right) \lambda_{k \mid k-1}^{u}\left(\mathbf{x}_{k}\right) .
$$

The number of Bernoulli components is

$$
I_{k}=I_{k-1}+M_{k} \text {. }
$$

The association vector at time $k$ can be represented as $\mathbf{b}=\left(b_{1}, \cdots, b_{I_{k}}\right)$ where $b_{j} \in\left\{1, \cdots, I_{k-1}\right\}$ indexes the track associated with measurement $j$ for $j \in\left\{1, \cdots, M_{k}\right\}$. Here, $b_{j}=0$ indicates that the new target is associated with measure- 
ment $j$. Furthermore, for $j=M_{k}+i$ with $i \in\left\{1, \cdots, I_{k-1}\right\}$, $b_{j}=i$ indicates that target $i$ is associated with no measurement. For the new tracks and existing tracks $j \in\left\{1, \cdots, M_{k}\right\}$, the association weights are given by

$$
w_{k}^{0, j}=\lambda_{c}\left(\mathbf{z}_{k}^{j}\right)+\int f\left(\mathbf{z}_{k}^{j} \mid \mathbf{x}_{k}\right) p_{d}\left(\mathbf{x}_{k}\right) \lambda_{k \mid k-1}^{u}\left(\mathbf{x}_{k}\right) \mathrm{d} \mathbf{x}_{k}
$$

and

$$
w_{k}^{b_{j}, j}=r_{k \mid k-1}^{b_{j}} \int f\left(\mathbf{z}_{k}^{j} \mid \mathbf{x}_{k}\right) p_{d}\left(\mathbf{x}_{k}\right) s_{k \mid k-1}^{b_{j}}\left(\mathbf{x}_{k}\right) \mathrm{d} \mathbf{x}_{k}
$$

for $b_{j} \in\left\{1, \cdots, I_{k-1}\right\}$; the existence probabilities are given by

$$
r_{k}^{0, j}=\frac{\int f\left(\mathbf{z}_{k}^{j} \mid \mathbf{x}_{k}\right) p_{d}\left(\mathbf{x}_{k}\right) \lambda_{k \mid k-1}^{u}\left(\mathbf{x}_{k}\right) \mathrm{d} \mathbf{x}_{k}}{\lambda_{c}\left(\mathbf{z}_{k}^{j}\right)+\int f\left(\mathbf{z}_{k}^{j} \mid \mathbf{x}_{k}\right) p_{d}\left(\mathbf{x}_{k}\right) \lambda_{k \mid k-1}^{u}\left(\mathbf{x}_{k}\right) \mathrm{d} \mathbf{x}_{k}}
$$

and $r_{k}^{b_{j}, j}=1$ for $b_{j} \in\left\{1, \cdots, I_{k-1}\right\}$; and the spatial distributions are given by

$$
s_{k}^{0, j}\left(\mathbf{x}_{k}\right)=\frac{f\left(\mathbf{z}_{k}^{j} \mid \mathbf{x}_{k}\right) p_{d}\left(\mathbf{x}_{k}\right) \lambda_{k \mid k-1}^{u}\left(\mathbf{x}_{k}\right)}{\int f\left(\mathbf{z}_{k}^{j} \mid \mathbf{x}_{k}^{\prime}\right) p_{d}\left(\mathbf{x}_{k}^{\prime}\right) \lambda_{k \mid k-1}^{u}\left(\mathbf{x}_{k}^{\prime}\right) \mathrm{d} \mathbf{x}_{k}^{\prime}}
$$

and

$$
s_{k}^{b_{j}, j}\left(\mathbf{x}_{k}\right)=\frac{f\left(\mathbf{z}_{k}^{j} \mid \mathbf{x}_{k}\right) p_{d}\left(\mathbf{x}_{k}\right) s_{k \mid k-1}^{b_{j}}\left(\mathbf{x}_{k}\right)}{\int f\left(\mathbf{z}_{k}^{j} \mid \mathbf{x}_{k}^{\prime}\right) p_{d}\left(\mathbf{x}_{k}^{\prime}\right) s_{k \mid k-1}^{b_{j}}\left(\mathbf{x}_{k}^{\prime}\right) \mathrm{d} \mathbf{x}_{k}^{\prime}}
$$

for $b_{j} \in\left\{1, \cdots, I_{k-1}\right\}$.

For missed tracks $j=M_{k}+i$ with $i \in\left\{1, \cdots, I_{k-1}\right\}$ and $b_{j}=\{0, i\}$, the association weights are given by

$$
w_{k}^{i, j}=1-r_{k \mid k-1}^{i}+r_{k \mid k-1}^{i} \int\left(1-p_{d}\left(\mathbf{x}_{k}\right)\right) s_{k \mid k-1}^{i}\left(\mathbf{x}_{k}\right) \mathrm{d} \mathbf{x}_{k}
$$

and $w_{k}^{0, j}=1$; the existing probabilities are given by $r_{k}^{0, j}=0$ and

$$
r_{k}^{i, j}=\frac{r_{k \mid k-1}^{i} \int\left(1-p_{d}\left(\mathbf{x}_{k}\right)\right) s_{k \mid k-1}^{i}\left(\mathbf{x}_{k}\right) \mathrm{d} \mathbf{x}_{k}}{1-r_{k \mid k-1}^{i}+r_{k \mid k-1}^{i} \int\left(1-p_{d}\left(\mathbf{x}_{k}\right)\right) s_{k \mid k-1}^{i}\left(\mathbf{x}_{k}\right) \mathrm{d} \mathbf{x}_{k}}
$$

and the spatial distributions are given by

$$
s_{k}^{i, j}\left(\mathbf{x}_{k}\right)=\frac{\left(1-p_{d}\left(\mathbf{x}_{k}\right)\right) s_{k \mid k-1}^{i}\left(\mathbf{x}_{k}\right)}{\int\left(1-p_{d}\left(\mathbf{x}_{k}^{\prime}\right)\right) s_{k \mid k-1}^{i}\left(\mathbf{x}_{k}^{\prime}\right) \mathrm{d} \mathbf{x}_{k}^{\prime}} .
$$

$\mathbf{p}_{k}$ is the marginal association probability at time $k$, which is approximated efficiently by the LBP algorithm based on the association weights $\mathbf{w}_{k}$. For the missed detection hypotheses $j=M_{k}+i$ with $i \in\left\{1, \cdots, I_{k-1}\right\}$,

$$
\begin{gathered}
r_{k}^{i}=p_{k}^{j}(i) r_{k}^{i, j}, \\
s_{k}^{i}\left(\mathbf{x}_{k}\right)=s_{k}^{i, j}\left(\mathbf{x}_{k}\right) .
\end{gathered}
$$

For the hypotheses of the existing tracks and new tracks $i=I_{k-1}+j$ with $j \in\left\{1, \cdots, M_{k}\right\}$,

$$
\begin{gathered}
r_{k}^{i}=\sum_{h=0}^{I_{k-1}} p_{k}^{j}(h) r_{k}^{h, j}, \\
s_{k}^{i}\left(\mathbf{x}_{k}\right)=\frac{1}{r_{k}^{i}} \sum_{h=0}^{I_{k-1}} p_{k}^{j}(h) r_{k}^{h, j} s_{k}^{h, j}\left(\mathbf{x}_{k}\right) .
\end{gathered}
$$

\subsection{Problem Description}

In the MOMB/P filter, the missed detection hypothesis probability in marginal association probability will be exaggerated when the target is detected. This is due to the fact that measurement information is not used in the missed detection hypotheses, as seen in (16). Therefore, the target may be lost since the detection hypothesis probability is small.

To solve this problem, measurement information should be used in the missed detection hypotheses. This results in a more accurate marginal association probability to make the cardinality estimation more accurate.

At the same time, an important situation should be attended. If the measurement is used in the missed detection hypothesis probabilities, the measurements of detected targets will interfere the missed detection hypothesis probabilities of missed targets when targets are together as close as possible. The cardinality estimation exhibits great bias in such situation. Thus, it is necessary to judge this situation where targets are close with each other.

\section{IMOMB/P Filter}

In this section, the solution to the problem in Sec. 2.3 is presented and the linear Gaussian implementation of the IMOMB/P filter is given.

\subsection{Problem Solution}

In the linear Gaussian implementation of the MOMB/P filter, suppose that the predicted multi-Bernoulli mixture contains $I_{k-1}$ Bernoulli components at time $k-1$, each composes a probability of existence $r_{k \mid k-1}^{i}$, a mean $\overline{\mathbf{x}}_{k \mid k-1}^{i}$ and covariance $\mathbf{P}_{k \mid k-1}^{i}$ as follow

$$
\left\{\left(r_{k \mid k-1}^{i}, \overline{\mathbf{x}}_{k \mid k-1}^{i}, \mathbf{P}_{k \mid k-1}^{i}\right)\right\}_{i=1}^{I_{k-1}} .
$$

The association weight $w_{k}^{i, j}$, for $j=M_{k}+i$ with $i \in\left\{1, \cdots, I_{k-1}\right\}$, describes the probability that target $i$ is associated with no measurement. When targets are well separated, it is unreasonable that $w_{k}^{i, j}$ is large for $j=M_{k}+i$ with $i \in\left\{1, \cdots, I_{k-1}\right\}$, even if there are one or more measurements close to the target state. In order to get more accurate missed detection hypothesis probability in marginal association probability of well separated targets, $w_{k}^{i, j}$ is modified with $g\left(\overline{\mathbf{z}}_{k}^{i} \mid \mathbf{x}_{k}\right)$, describing missed detection hypotheses, where $\overline{\mathbf{z}}_{k}^{i}$ is the nearest measurement to the target state $\overline{\mathbf{x}}_{k \mid k-1}^{i}$. Thus, $w_{k}^{i, j}$ is rewritten as

$$
w_{k}^{i, j}=1-r_{k \mid k-1}^{i}+r_{k \mid k-1}^{i} \int g\left(\overline{\mathbf{z}}_{k}^{i} \mid \mathbf{x}_{k}\right)\left(1-p_{d}\left(\mathbf{x}_{k}\right)\right) s_{k \mid k-1}^{i}\left(\mathbf{x}_{k}\right) \mathrm{d} \mathbf{x}_{k} .
$$

The likelihood function $f\left(\mathbf{z}_{k} \mid \mathbf{x}_{k}\right)$ is generally a Gaussian distribution which describes the possibility that the measurement $\mathbf{z}_{k}$ is generated by the target state $\mathbf{x}_{k}$. In addition, $g\left(\overline{\mathbf{z}}_{k}^{i} \mid \mathbf{x}_{k}\right)$ describes the influence of the measure- 
ment $\overline{\mathbf{z}}_{k}^{i}$ to the target state $\mathbf{x}_{k}$ when the target is hypothesized to be missed. When the measurement $\overline{\mathbf{z}}_{k}^{i}$ is close to the target state $\mathbf{x}_{k}, g\left(\overline{\mathbf{z}}_{k}^{i} \mid \mathbf{x}_{k}\right)$ should have a small value which means the target is unlikely missed because the measurement $\overline{\mathbf{z}}_{k}^{i}$ may be generated by the target. On the contrary, when the measurement $\overline{\mathbf{z}}_{k}^{i}$ is far away from the target state $\mathbf{x}_{k}, g\left(\overline{\mathbf{z}}_{k}^{i} \mid \mathbf{x}_{k}\right)$ should have a big value which means the target may be missed because the measurement $\overline{\mathbf{z}}_{k}^{i}$ is unlikely generated by the target. It is worth noting that the value of $g\left(\overline{\mathbf{z}}_{k}^{i} \mid \mathbf{x}_{k}\right)$ should have the same magnitude as $f\left(\mathbf{z}_{k} \mid \mathbf{x}_{k}\right)$. Therefore, $g\left(\overline{\mathbf{z}}_{k}^{i} \mid \mathbf{x}_{k}\right)$ is defined as an exponential function, as follows

$$
g\left(\overline{\mathbf{z}}_{k}^{i} \mid \mathbf{x}_{k}\right)=\frac{1}{\sqrt{2 \pi}} \exp \left[-\frac{2 \mathbf{P}_{k \mid k-1}^{i}}{\left(\mathbf{H} \overline{\mathbf{x}}_{k \mid k-1}^{i}-\overline{\mathbf{z}}_{k}^{i}\right)^{T}\left(\mathbf{H} \overline{\mathbf{x}}_{k \mid k-1}^{i}-\overline{\mathbf{z}}_{k}^{i}\right)}\right] .
$$

By (24) and (25), $w_{k}^{i, j}$ is smaller when there are one or more measurements close to the target state. It is more reasonable that missed detection occurs only when no measurement is around the target. Thus, the marginal association probability is more accurate based on $w_{k}^{i, j}$. In the proposed filter, the other parts are almost the same as the MOMB/P filter except $w_{k}^{i, j}$.

\subsection{The IMOMB/P Filter}

As mentioned in Sec. 2.3, the situation should be noticed where targets are close. To handle this situation, a judge step in the proposed filter is required. Then, the corresponding linear Gaussian implementation of the $\mathrm{IMOMB} / \mathrm{P}$ filter is summarized in this section.

Firstly, the prediction step is the same as that of the MOMB/P filter.

Secondly, the judge step is given as follows. Suppose that the estimated tracks are $\left\{\left(\hat{r}_{k-1}^{i}, \hat{\mathbf{x}}_{k-1}^{i}, \hat{\mathbf{P}}_{k-1}^{i}\right)\right\}_{i=1}^{\left|\hat{\mathbf{x}}_{k-1}\right|}$ at time $k-1$. If there exist $\hat{\mathbf{x}}_{k-1}^{i}$ and $\hat{\mathbf{x}}_{k-1}^{j} \quad(i \neq j)$ satisfying $\left|\hat{\mathbf{x}}_{k-1}^{i}-\hat{\mathbf{x}}_{k-1}^{j}\right|<\left|\hat{\mathbf{P}}_{k-1}^{i}\right|^{\frac{1}{2}}+\left|\hat{\mathbf{P}}_{k-1}^{j}\right|^{\frac{1}{2}}$, the update step same to that of the $\mathrm{MOMB} / \mathrm{P}$ filter is performed. Otherwise, go to the update step.

Then, the update step is given as follows. Suppose that at time $k$, the predicted intensity of the unknown targets is a Gaussian mixture as follows

$$
\lambda_{k \mid k-1}^{u}=\sum_{i=1}^{I_{k-1}^{u}} \lambda_{k \mid k-1}^{u, i} \mathcal{N}\left(\mathbf{x} ; \overline{\mathbf{x}}_{k \mid k-1}^{u, i}, \mathbf{P}_{k \mid k-1}^{u, i}\right)
$$

where $\mathcal{N}(\mathbf{x} ; \mathbf{m}, \mathbf{P})$ denotes a multivariate Gaussian distribution defined over the variable vector $\mathbf{x}$ with mean vector $\mathbf{m}$ and covariance matrix $\mathbf{P}$. Additionally, the predicted multi-Bernoulli mixture contains $I_{k-1}$ Bernoulli components, each composes a probability of existence $r_{k \mid k-1}^{i}$, a mean $\overline{\mathbf{x}}_{k \mid k-1}^{i}$ and covariance $\mathbf{P}_{k \mid k-1}^{i}$ as follows

$$
\left\{\left(r_{k \mid k-1}^{i}, \overline{\mathbf{x}}_{k \mid k-1}^{i}, \mathbf{P}_{k \mid k-1}^{i}\right)\right\}_{i=1}^{I_{k-1}} .
$$

The updated intensity of the unknown targets is

$$
\lambda_{k}^{u}=\sum_{i=1}^{I_{k}^{u}} \lambda_{k}^{u, i} \mathcal{N}\left(x ; \overline{\mathbf{x}}_{k}^{u, i}, \mathbf{P}_{k}^{u, i}\right)
$$

where $\quad \lambda_{k}^{u, i}=\left(1-p_{d}\right) \lambda_{k \mid k-1}^{u, i} \quad, \quad \overline{\mathbf{x}}_{k}^{u, i}=\overline{\mathbf{x}}_{k \mid k-1}^{u, i}, \quad \mathbf{P}_{k}^{u, i}=\mathbf{P}_{k \mid k-1}^{u, i} \quad$ and $I_{k}^{u}=I_{k-1}^{u}$.

Here, the updated multi-Bernoulli mixture with $I_{k}$ Bernoulli components can be divided into two parts as follows

$$
\left\{\left(r_{k}^{i}, \overline{\mathbf{x}}_{k}^{i}, \mathbf{P}_{k}^{i}\right)\right\}_{i=1}^{I_{k-1}}
$$

and

$$
\left\{\left(r_{k}^{i}, \overline{\mathbf{x}}_{k}^{i}, \mathbf{P}_{k}^{i}\right)\right\}_{i=I_{k-1}+1}^{I_{k}}
$$

where $I_{k}=I_{k-1}+M_{k}$.

In the first part, i.e., (29), for missed tracks $j=M_{k}+i$ with $i \in\left\{1, \cdots, I_{k-1}\right\}$,

$$
\begin{gathered}
r_{k}^{i}=p_{k}^{j}(i) r_{k}^{i, j}, \\
r_{k}^{i, j}=\frac{r_{k \mid k-1}^{i}\left(1-p_{d}\right)}{1-r_{k \mid k-1}^{i}+r_{k \mid k-1}^{i}\left(1-p_{d}\right)} \\
\overline{\mathbf{x}}_{k}^{i}=\overline{\mathbf{x}}_{k \mid k-1}^{i}, \\
\mathbf{P}_{k}^{i}=\mathbf{P}_{k \mid k-1}^{i}
\end{gathered}
$$

where $p_{k}^{j}(i)$ is the marginal association probability.

In the second part, i.e., (30), for existing tracks and new tracks $i=I_{k-1}+j$ with $j \in\left\{1, \cdots, M_{k}\right\}$,

$$
\begin{gathered}
r_{k}^{i}=\sum_{h=0}^{I_{k-1}} p_{k}^{j}(h) r_{k}^{h, j}, \\
\overline{\mathbf{x}}_{k}^{i}=\frac{1}{r_{k}^{i}} \sum_{h=0}^{I_{k-1}} p_{k}^{j}(h) r_{k}^{h, j} \overline{\mathbf{x}}_{k}^{h, j}, \\
\mathbf{P}_{k}^{i}=\frac{1}{r_{k}^{i}} \sum_{h=0}^{I_{k-1}} p_{k}^{j}(h) r_{k}^{h, j}\left(\mathbf{P}_{k}^{h, j}+\left(\overline{\mathbf{x}}_{k}^{h, j}-\overline{\mathbf{x}}_{k}^{i}\right)\left(\overline{\mathbf{x}}_{k}^{h, j}-\overline{\mathbf{x}}_{k}^{i}\right)^{\mathrm{T}}\right)
\end{gathered}
$$

where

$$
\begin{gathered}
r_{k}^{h, j}=1, \\
\overline{\mathbf{x}}_{k}^{h, j}=\overline{\mathbf{x}}_{k \mid k-1}^{h}+\mathbf{K}\left(\mathbf{z}_{k}^{j}-\mathbf{H} \overline{\mathbf{x}}_{k \mid k-1}^{h}\right), \\
\mathbf{P}_{k}^{h, j}=\mathbf{P}_{k \mid k-1}^{h}-\mathbf{K} \mathbf{H} \mathbf{P}_{k \mid k-1}^{h}, \\
\mathbf{K}=\mathbf{P}_{k \mid k-1}^{h} \mathbf{H}^{\mathrm{T}}\left(\mathbf{H} \mathbf{P}_{k \mid k-1}^{h} \mathbf{H}^{\mathrm{T}}+\mathbf{R}\right)^{-1}
\end{gathered}
$$

and $\mathbf{H}$ is the measurement matrix for $h \in\left\{1, \cdots, I_{k-1}\right\}$;

$$
r_{k}^{0, j}=\frac{C}{w_{k}^{0, j}},
$$




$$
\begin{gathered}
\overline{\mathbf{x}}_{k}^{0, j}=\frac{1}{C} \sum_{s=1}^{I_{k-1}^{u}} c_{s} \mathbf{y}_{s}, \\
\mathbf{P}_{k}^{0, j}=\frac{1}{C} \sum_{s=1}^{I_{k-1}^{u}} c_{s}\left[\mathbf{P}_{s}+\left(\overline{\mathbf{x}}_{k}^{0, j}-\mathbf{y}_{s}\right)\left(\overline{\mathbf{x}}_{k}^{0, j}-\mathbf{y}_{s}\right)^{\mathrm{T}}\right] \\
\mathbf{y}_{s}=\overline{\mathbf{x}}_{k \mid k-1}^{u, s}+\mathbf{K}_{s} \mathbf{v}, \\
C=\sum_{s=1}^{I_{k-1}^{u}} c_{s}, \\
c_{s}=\lambda_{k \mid k-1}^{u, s} p_{d} \mathcal{N}\left(\mathbf{v} ; \mathbf{0}, \mathbf{S}_{s}\right), \\
\mathbf{v}=\mathbf{z}_{k}^{i}-\mathbf{H} \overline{\mathbf{x}}_{k \mid k-1}^{u, s}, \\
\mathbf{S}_{s}=\mathbf{H} \mathbf{P}_{k \mid k-1}^{u, s} \mathbf{H}^{\mathrm{T}}+\mathbf{R}, \\
\mathbf{K}_{s}=\mathbf{P}_{k \mid k-1}^{u, s} \mathbf{H}^{\mathrm{T}}\left(\mathbf{S}_{s}\right)^{-1}, \\
\mathbf{P}_{s}=\mathbf{P}_{k \mid k-1}^{u, s}-\mathbf{K}_{s} \mathbf{H} \mathbf{P}_{k \mid k-1}^{u, s} .
\end{gathered}
$$

The marginal association probabilities $\mathbf{p}_{k}$ at time $k$ can be computed by the LBP algorithm according to the association weights $\mathbf{w}_{k}$, namely,

$$
w_{k}^{i, j}=1-r_{k \mid k-1}^{i}+r_{k \mid k-1}^{i}\left(1-p_{d}\right) g\left(\overline{\mathbf{z}}_{k}^{i}\right)
$$

for $j=M_{k}+i$ with $i \in\left\{1, \cdots, I_{k-1}\right\}$, and

$$
\begin{gathered}
w_{k}^{0, j}=C+\lambda^{f a}, \\
w_{k}^{b_{j}, j}=r_{k \mid k-1}^{b_{j}} p_{d} \mathcal{N}\left(\mathbf{v} ; \mathbf{0}, \mathbf{S}_{b_{j}}\right), \\
\mathbf{v}=\mathbf{z}_{k}^{j}-\mathbf{H x}_{k \mid k-1}^{b_{j}}, \\
\mathbf{S}_{b_{j}}=\mathbf{H P}_{k \mid k-1}^{b_{j}} \mathbf{H}+\mathbf{R}
\end{gathered}
$$

for $j \in\left\{1, \cdots, M_{k}\right\}$ and $b_{i} \in\left\{1, \cdots, I_{k-1}\right\}$.

Finally, pruning and estimation are needed to reduce the computational burden. Only MB components with $r_{k}^{j}$ larger than a threshold $\theta_{t h}^{p}$ (e.g. $10^{-4}$ in [12]) are retained in the next prediction step. Meanwhile, a MB component is considered to exist at time $k$ when $r_{k}^{j}$ is large than a threshold $\theta_{t h}^{d}$ (e.g. 0.8 in [12]). Then, the estimation $\hat{\mathbf{X}}_{k}$ at time $k$ can be obtained.

\section{Simulation Results}

The performance of the IMOMB/P filter is demonstrated in a challenging scenario where six targets cross each other in a certain region. The true trajectories are shown in Fig. 1. The sampling period $T$ is set to be $1 \mathrm{~s}$, and targets follow a linear Gaussian model

$$
\mathbf{x}_{k}=\mathbf{F} \mathbf{x}_{k-1}+\mathbf{w}_{k}
$$

where $\mathbf{x}_{k}=\left[p_{x, k}, \dot{p}_{x, k}, p_{y, k}, \dot{p}_{y, k}\right]^{\mathrm{T}}$ consists of the position and velocity of a target at time $k$ and $\mathbf{w}_{k} \sim \mathcal{N}\{\mathbf{0}, \mathbf{Q}\} . \mathbf{F}$ and $\mathbf{Q}_{k}$ are computed by

$$
\begin{gathered}
\mathbf{F}=\left[\begin{array}{ll}
1 & T \\
0 & 1
\end{array}\right] \otimes \mathbf{I}_{2 \times 2}, \\
\mathbf{Q}_{k}=q\left[\begin{array}{cc}
T^{3} / 3 & T^{2} / 2 \\
T^{2} / 2 & T
\end{array}\right] \otimes \mathbf{I}_{2 \times 2}
\end{gathered}
$$

where $q=0.01$. It is assumed that the position of a target is observed with a Gaussian noise

$$
\mathbf{z}_{k}=\mathbf{H} \mathbf{x}_{k}+\mathbf{v}_{k}
$$

where $\mathbf{H}=\left[\begin{array}{ll}1 & 0\end{array}\right] \otimes \mathbf{I}_{2 \times 2}$ and $\mathbf{v}_{k} \sim \mathcal{N}\left(\mathbf{0}, \mathbf{I}_{2 \times 2}\right)$. The intensity function for undetected targets is assumed to be $\lambda_{u}\left(\mathbf{x}_{k}\right) \sim 10 \mathcal{N}(\mathbf{0}, \mathbf{P})$, where $\mathbf{P}=\operatorname{diag}\left[100^{2}, 1,100^{2}, 1\right]$. The birth intensity function is $\lambda_{b}\left(\mathbf{x}_{k}\right) \sim 0.05 \mathcal{N}(\mathbf{0}, \mathbf{P})$, where the birth intensity uses the same covariance in $\lambda_{u}\left(\mathbf{x}_{k}\right) \cdot p_{s}\left(\mathbf{x}_{k}\right)$ is set to be 0.999 . In this work, only the cases with $p_{d}\left(\mathbf{x}_{k}\right) \in\{0.5,0.7,0.9\}$ and $\lambda_{c}\left(\mathbf{z}_{k}\right) \in\{10,50\}$ are considered.

The Optimal Subpattern assignment (OSPA) distance [17] is used to evaluate the performance of the tracking algorithms which is defined as

$$
\bar{d}_{p}^{c}(\mathbf{X}, \mathbf{Y})=\left[\frac{1}{n}\left(\min _{\pi \in \Pi_{n}} \sum_{i=1}^{m} d^{c}\left(\mathbf{x}_{i}, \mathbf{y}_{\pi_{i}}\right)^{p}+c^{p}(n-m)\right)\right]^{1 / p}
$$

where $\mathbf{X}=\left\{\mathbf{x}_{1}, \cdots, \mathbf{x}_{m}\right\}$ and $\mathbf{Y}=\left\{\mathbf{y}_{1}, \cdots, \mathbf{y}_{n}\right\}$ are finite subsets. $p$ and $c$ are set to be 2 and 100, respectively. The simulations are implemented by MATLAB R2015b on Intel Core i3-7100 3.90 GHz processor and 8 GB RAM.

To verify the validation of the proposed filter, 200 Monte Carlo trails with different $p_{d}$ and $\lambda_{c}$ are performed. The OSPA distances of the MOMB/P and IMOMB/P filters are shown in Fig. 2 where logarithmic coordinate system is used to get the difference distinctly. From the results, it can be seen that the IMOMB/P filter outperforms the $\mathrm{MOMB} / \mathrm{P}$ filter in most cases. The reason is that the IMOMB/P filter has more accurate missed detection hypothesis probabilities in the marginal association probabilities, which allows measurement information be normally used in the update step. At the same time, both $\mathrm{MOMB} / \mathrm{P}$ filter and IMOMB/P filter can clearly response to cardinality changes and are robust to the coalescence. During the long time coalescence, the OSPA distance of these two filters are almost the same. This is because the $\mathrm{MOMB} / \mathrm{P}$ update is performed by the judge step. When $p_{d}$ is 0.9, as shown in Figs. 2(e) and 2(f), these two filters almost have same performance and can both track well. It is because that the missed detection hypothesis probability in the marginal association probability is small with high $p_{d}$, which is close to the accurate missed detection hypothesis probability. Compared to the MOMB/P filter, the proposed filter perfects much better in cases with $p_{d}=0.5$ and $p_{d}=0.7$. Hence, the IMOMB/P filter improves upon the performance of the $\mathrm{MOMB} / \mathrm{P}$ filter, especially with lower $p_{d}$ values. When $p_{d}$ is fixed and $\lambda_{c}$ increases from 10 to 50 , such as, Figs. 2(a) and 2(b) with $p_{d}=0.5$, both of them can achieve good tracking performance even though the OSPA 


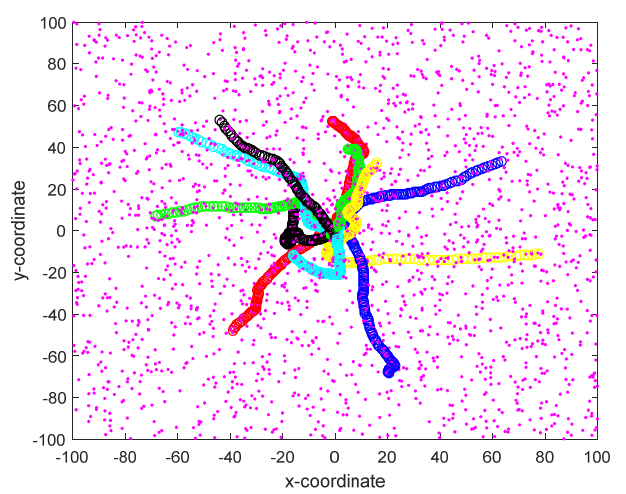

Fig. 1. True target trajectories with $p_{d}=0.7, \quad \lambda_{c}=10$. Trajectories are shown in small circles and measurements are shown in points.

distances of these two filters are both larger. It can be seen that the IMOMB/P filter is also robust to higher $\lambda_{c}$. When $p_{d}=0.7$ and $\lambda_{c}=50$, the average run time of each time step of the MOMB/P filter and the IMOMB/P filter are $0.0021 \mathrm{~s}$ and $0.0023 \mathrm{~s}$, respectively. These two filters are both efficient. It is due to the fact that they are based on the LBP algorithm with high efficiency. Although the computation efficiency of the IMOMB/P filter is slightly lower than that of the MOMB/P filter, it does not affect the real-time performance.

Figure 3 shows the cardinality estimation of these two filters where 500 Monte Carlo trails are performed with $p_{d}=0.7$ and $\lambda_{c}=10$. In Fig. 3(a), it can be seen that both $\mathrm{MOMB} / \mathrm{P}$ filter and IMOMB filter can response clearly to cardinality changes. To see the difference between the $\mathrm{MOMB} / \mathrm{P}$ filter and IMOMB/P filter, the partial enlarged detail of Fig. 3(a) is shown in Fig. 3(b) where the cardinality estimation of the proposed filter is closer to the true cardinality than that of the MOMB/P filter. The reason for this is that measurement information can be fully used in the update step to avoid the loss of tracking targets. Furthermore, the proposed filter has less bias in cardinality estimation than the MOMB/P filter, as shown in Fig. 3(c). Therefore, the IMOMB/P filter can improve the cardinality estimation accuracy of the MOMB/P filter.

\section{Conclusion}

Through the analysis the disadvantages of the $\mathrm{MOMB} / \mathrm{P}$ filter, the IMOMB/P filter has been proposed in this paper, which gives more accurate marginal association probabilities by considering measurement information in missed detection hypotheses. The simulation results have shown that the proposed filter can achieve better performance than the MOMB/P filter, especially with lower values of $p_{d}$. Although the proposed filter is robust to coalescence, it also has large error from the inaccurate association weights during the coalescence. This is because the covariance estimation of the target state is too large when it is interfered by the measurements of the others. The problem may be solved by increasing the number of iterations in the LBP algorithm. Possible direction for future research includes the problems in the coalescence.

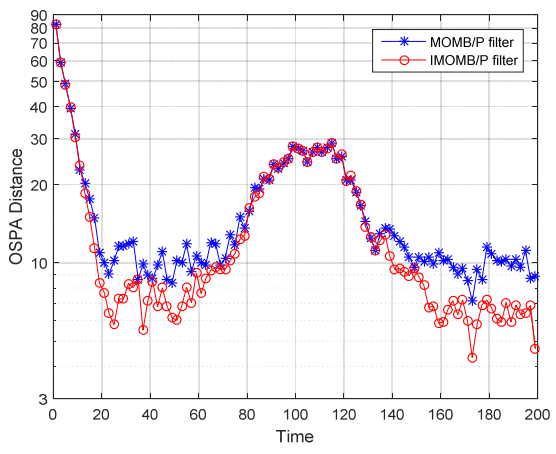

(a) $p_{d}=0.5, \lambda_{c}=10$

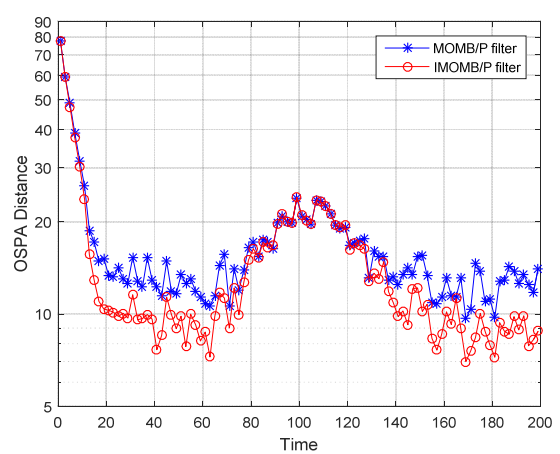

(d) $p_{d}=0.7, \lambda_{c}=50$

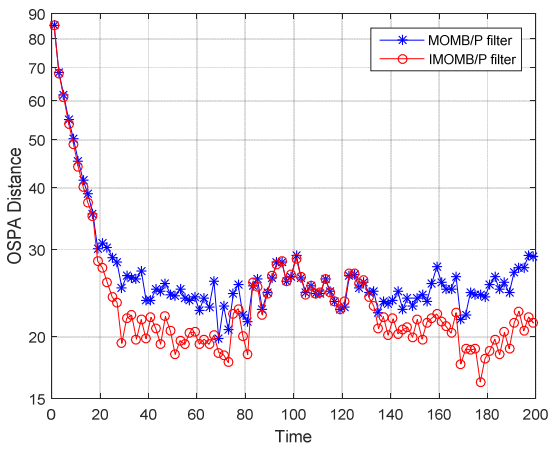

(b) $p_{d}=0.5, \lambda_{c}=50$

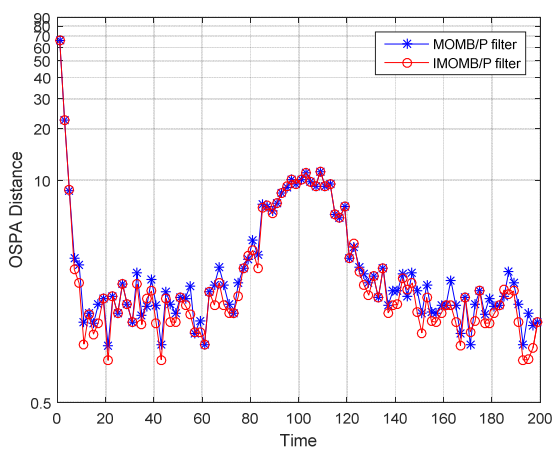

(e) $p_{d}=0.9, \lambda_{c}=10$

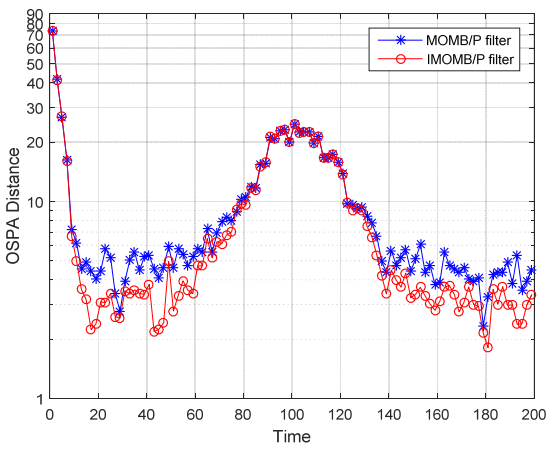

(c) $p_{d}=0.7, \lambda_{c}=10$

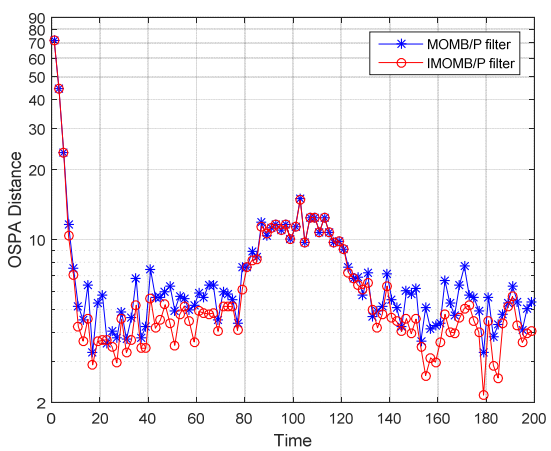

(f) $p_{d}=0.9, \lambda_{c}=50$

Fig. 2. OSPA distances of two different filters. 


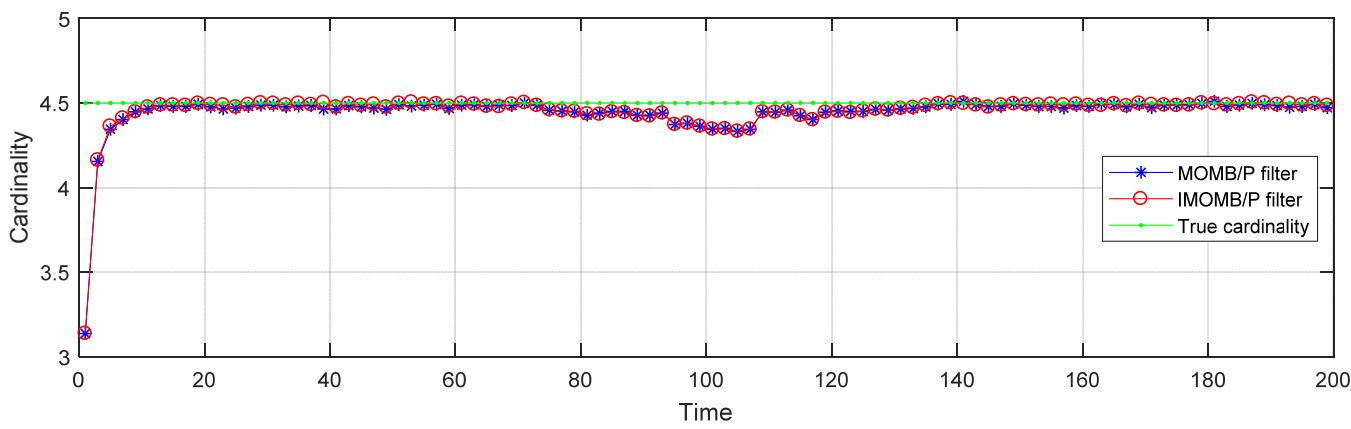

(a) Cardinality estimation.

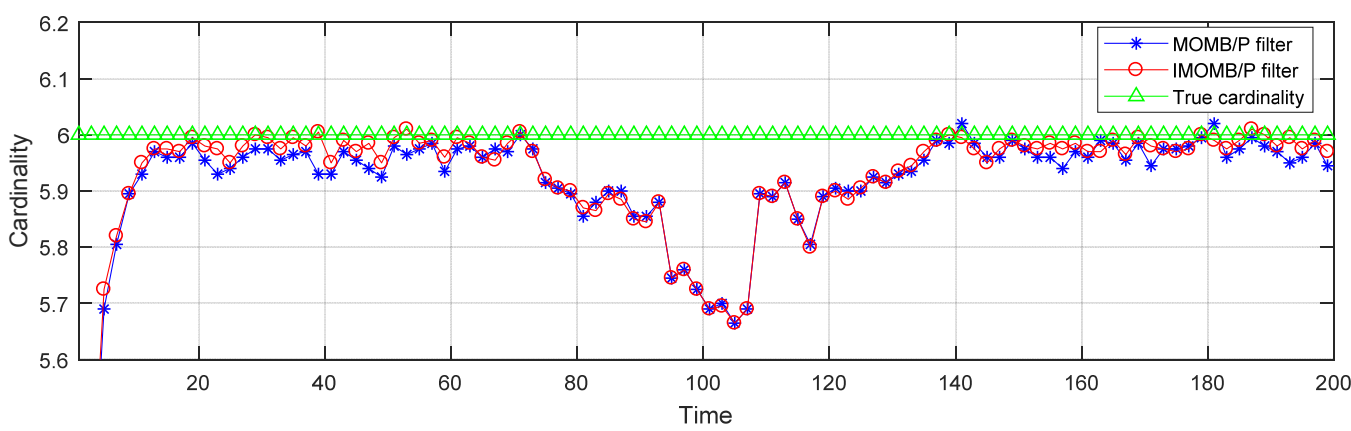

(b) Cardinality estimation.

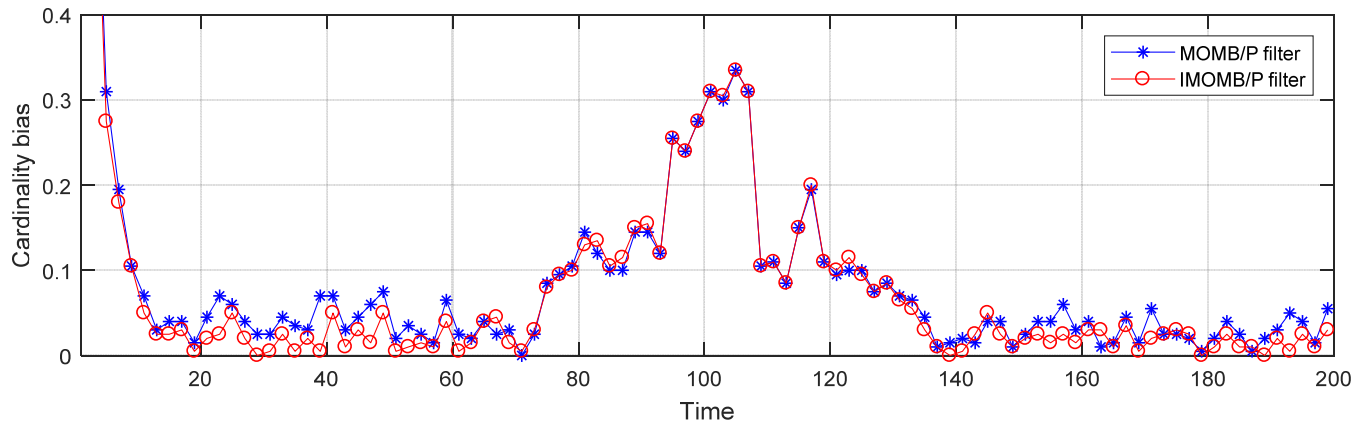

(c) The cardinality estimation of two different filters.

Fig. 3. The cardinality estimation of two different filters.

\section{Acknowledgments}

This work was supported by the National Natural Science Foundation of China under grant no. 61372003 and 61503293, the National Natural Science Foundation of Shaanxi Province under grant no. 2018JQ6059 and the Postdoctoral Science Foundation of China under grant no. 2018M633470.

\section{References}

[1] MAHLER, R. P. S. Statistical Multisource-Multitarget Information Fusion. Boston (USA): Artech House, 2007. ISBN: 15969309269781596930926

[2] MAHLER, R. P. S. Advances in Statistical MultisourceMultitarget Information Fusion. Boston (USA): Artech House, 2014. ISBN: 9781608077984

[3] MAHLER, R. P. S. Multitarget Bayes filtering via first-order multitarget moments. IEEE Transactions on Aerospace and
Electronic Systems, 2003, vol. 39, no. 4, p. 1152-1178. DOI: 10.1109/TAES.2003.1261119

[4] MAHLER, R. P. S. PHD filters of higher order in target number. IEEE Transactions on Aerospace and Electronic Systems, 2007, vol. 43, no. 4, p. 1523-1543. DOI: 10.1109/TAES.2007.4441756

[5] VO, B. T., VO, B. N., CANTONI, A. The cardinality balanced multi-target multi-Bernoulli filter and its implementations. IEEE Transactions on Signal Processing, 2009, vol. 57, no. 2, p. 409-423. DOI: 10.1109/TSP.2008.2007924

[6] VO, B. N., SINGH, S., DOUCET, A. Sequential Monte Carlo methods for multitarget filtering with random finite sets. IEEE Transactions on Aerospace and Electronic Systems, 2005, vol. 41, no. 4, p. 1224-1245. DOI: 10.1109/TAES.2005.1561884

[7] VO, B. T., VO, B. N., CANTONI, A. Analytic implementations of the cardinalized probability hypothesis density filter. IEEE Transactions on Signal Processing, 2007, vol. 55, no. 7, p. 3553-3567. DOI: 10.1109/TSP.2007.894241

[8] WILliAMS, J. L. Experiments with graphical model implementations of multiple target multiple Bernoulli filters. In Proceedings of the 11th Intelligent Sensors, Sensor Networks and Information Processing (ISSNIP). Adelaide (Australia), 2011, p. 532-537. DOI: 10.1109/ISSNIP.2011.6146620 
[9] VO, B. T., VO, B. N. Labeled random finite sets and multi-object conjugate priors. IEEE Transactions on Signal Processing, 2013, vol. 61 , no. 13, p. 3460-3475. DOI: 10.1109/TSP.2013.2259822

[10] VO, B. T., VO, B. N., PHUNG, D. Labeled random finite sets and the Bayes multi-target tracking filter. IEEE Transactions on Signal Processing, 2014, vol. 62, no. 24, p. 6554-6567. DOI: 10.1109/TSP.2014.2364014

[11] REUTER, S., VO, B. T., VO, B. N., et al. The labeled multiBernoulli filter. IEEE Transactions on Signal Processing, 2014, vol. 62, no. 12, p. 3246-3260. DOI: 10.1109/TSP.2014.2323064

[12] WILLIAMS, J. L. Marginal multi-Bernoulli filters: RFS derivation of MHT, JIPDA, and association-based MeMBer. IEEE Transactions on Aerospace and Electronic Systems, 2015, vol. 51, no. 3, p. 1664-1687. DOI: 10.1109/TAES.2015.130550

[13] WILLIAMS, J. L. An efficient, variational approximation of the best fitting multi-Bernoulli filter. IEEE Transactions on Signal Processing, 2015, vol. 63, no. 1, p. 258-273. DOI: 10.1109/TSP.2014.2370946

[14] KROPFREITER, T., MEYER, F., HLAWATSCH, F. Sequential Monte Carlo implementation of the track-oriented marginal multiBernoulli/Poisson filter. In Proceedings of the 16th Information Fusion (FUSION). Heidelberg (Germany), 2016, p. 972-979. ISBN: 9780996452748

[15] GRANSTRÖM, K., FATEMI, M., SVENSSON, L. Gamma Gaussian inverse-Wishart Poisson multi-Bernoulli filter for extended target tracking. In Proceedings of the 16th Information Fusion (FUSION). Heidelberg (Germany), 2016, p. 513-520. ISBN: 9780996452748

[16] WILLIAMS, J. L., LAU, R. Approximate evaluation of marginal association probabilities with belief propagation. IEEE Transactions on Aerospace and Electronic Systems, 2014, vol. 50, no. 4, p. 2942-2959. DOI: 10.1109/TAES.2014.120568

[17] SCHUHMACHER, D., VO, B. T., VO, B. N. A consistent metric for performance evaluation of multi-object filters. IEEE Transactions on Signal Processing, 2008, vol. 56, no. 8, p. 3447-3457. DOI: 10.1109/TSP.2008.920469

\section{About the Authors ...}

Zhen-zhen SU was born in Shanxi, China in 1993. He received his B.S. degree in Applied Mathematics from Xidian University in 2015. He is currently a Ph.D. candidate at Xidian University. His research interests include target tracking, visual tracking and graphical models.

Hong-bing JI (corresponding author) was born in Shaanxi, China in 1963. He received the B.S. degree in Radar Engineering, M.S. degree in Circuit, Signals and Systems and his Ph.D. degree in Signal and Information Processing from Xidian University, Xi'an China, in 1983, 1988, and 1999, respectively. Since graduation in 1988, he has been with the School of Electronic Engineering at Xidian University, a lecturer from 1990 to 1995, an associate professor from 1995 to 2000, a professor since 2000, and an advisor for Ph.D. students since 2001. From 1996 to 2002 he served as a Vice Dean of the School of Electronic Engineering. From 2002 to 2006 he was the Executive Dean of the Graduate School at Xidian University. From 2006 to 2012, he served as the Director of the Development and Planning Department at Xidian University. He is currently the Dean of the Graduate School at Xidian University. His primary areas of research are radar signal processing, automatic target recognition, target tracking.

Yong-quan ZHANG recieved his B.S. degree and M.S. degree in Computer Science and Technology from Lanzhou University of Technology in 2007 and 2010. He received his $\mathrm{Ph} . \mathrm{D}$. degree in Intelligent Information Processing from Xidian University in 2014. He is currently a lecturer at Xidian University. His primary areas of research are target tracking and automatic target recognition. 